\title{
WAPM-Newsletter No 1/2010
}

\section{Report of the 9th World Congress of Perinatal Medicine, Berlin (Germany), October 24-28, 2009}

About 2200 participants met in the Estrel Convention Center in Berlin on the occasion of the $9^{\text {th }}$ World Congress of Perinatal Medicine, to discuss the hot topics of Perinatal Medicine.

During the opening ceremony the slogan "Take responsibility for the future" was already being discussed. The global problems of obstetrics and neonatology were put in perspective by the President of the congress, Joachim W. Dudenhausen as well as by the President of the World Association of Perinatal Medicine and by Lord Patel from Great Britain. Its main concerns were maternal death during pregnancy and childbirth as well as risk to the health of mother and child caused by unattended childbirth all around the world. The reasons for high maternal and fetal death rates are primarily low education

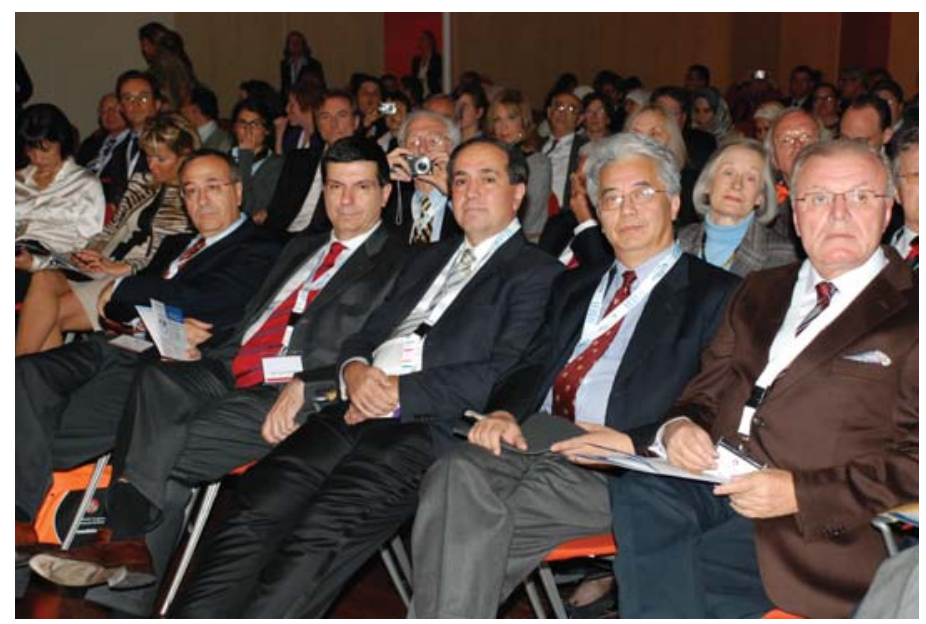

From left to right: Prof. Asim Kurjak, Prof. José Carrera, Prof. Frank A. Chervenak and Prof. Giancarlo DiRenzo. and poverty. Thus the speakers appealed to the persons in charge, to decrease these risks by better education and by measures to improve health care of women during pregnancy and birth.

A podium discussion enlightening high maternal death rates in different regions of the world was dedicated to this global aspect as well as another discussion dealing with maternal-fetal transmission of HIV. The risk of a perinatal infection by maternal HIV could almost entirely be prevented, provided that the mother and child are treated during pregnancy and birth. As several speakers from the WHO pointed out, devastating situations in different parts of the world could be solved with these measures.

The ethical aspects of Perinatology were also discussed, beginning with prenatal diagnosis and treatment of children at the limits of viability. Different points of view from different cultures were clarified in this context, but also the differ-

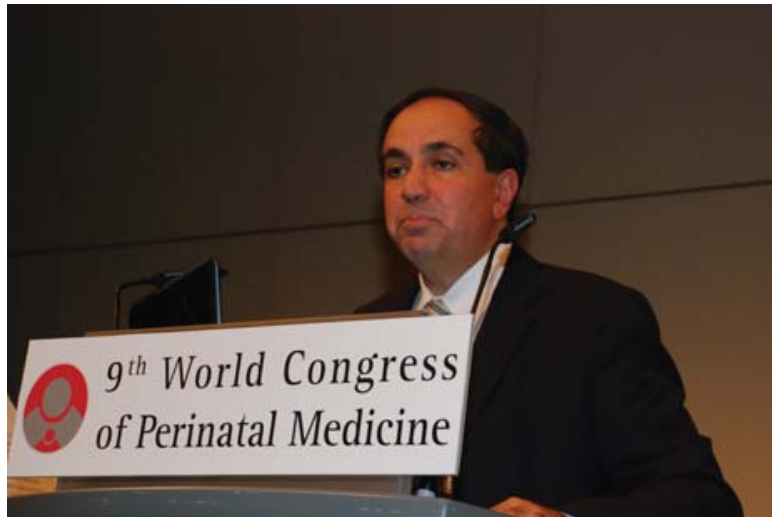

Frank A. Chervenak, The President of the World Association of Perinatal Medicine at the opening ceremony.

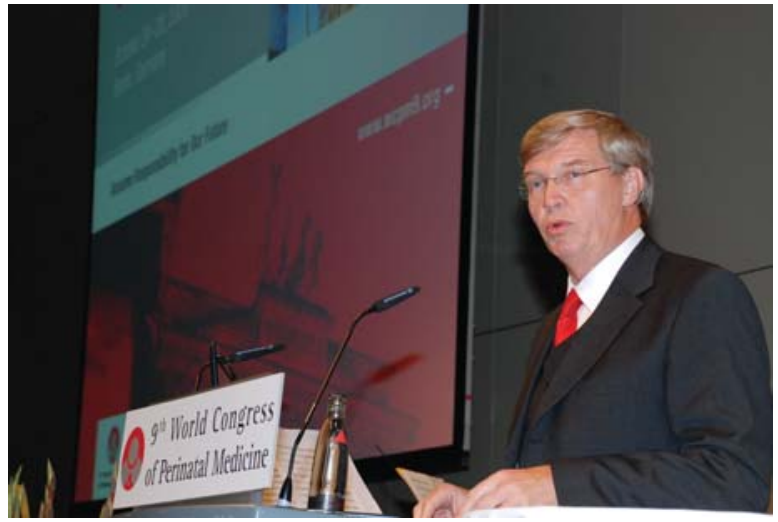

Joachim W. Dudenhausen, the Congress President at the opening ceremony. 


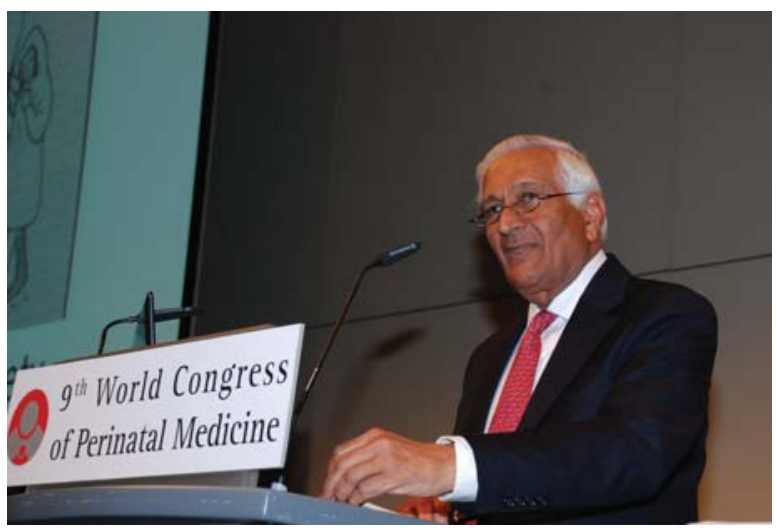

Lord Patel, Chancellor of the University of Dundee/Great Britain.

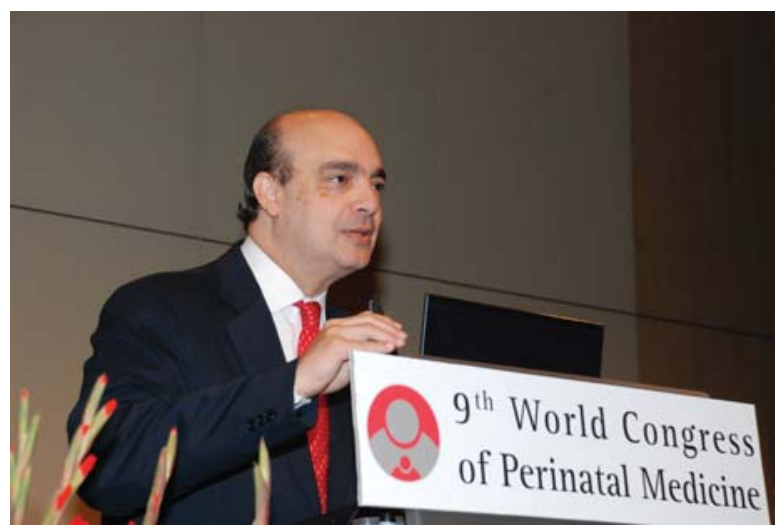

Roberto Romero from the Perinatology Research Branch, and Center for Molecular Medicine and Genetics, Wayne State University/USA.

ences within Europe were striking. The German position is extremely sensitive in comparison to the Netherlands or in Switzerland. The different approach was elaborated by participants from Australia and America. The Japanese position had changed significantly during the last years.

The discussion of the global importance of preterm labor was wide-ranging, and appropriate measures to prevent and manage preterm labor as well as treatment of preterm infants dominated essential parts of the congress. Roberto Romero from the US National Institute of Health introduced molecular diagnostics of preterm labor and provided insight into this fascinating future field that might allow for completely new techniques, and for the study of the modification of the genome, the transcriptome, the proteome and metabolome. We are sure that this new division of biology will create important advances regarding preterm labor within the next few years.

The attendance of many young scientists who presented their findings in free communications and poster presentations was very gratifying. The congress administration offered awards for different fields of posters and communications, and a core system assigned by the chairmen of the sessions allowed a transparent assessment of the outcomes. Finally, two awards could be given to lectures and three awards to poster sessions. Young scientists from Portugal, Great Britain, Korea, Germany and Japan were decorated and obtained the awards in the closing ceremony.

Joachim W. Dudenhausen Congress President

\section{Report of the President}

The WAPM Congress in Berlin was a success in every regard. With over 2000 international participants and a scientific program that featured world leaders in perinatal medicine, the numerous sessions were filled with state-of-the-art information and active interchanges among the participating perinatologists. The scope of the program was exceeded only by the depth and the originality of the presentations which left the participants better equipped to care for their maternal, fetal, and neonatal patients.

The scientific program was matched by the hospitality of Professor Joachim Dudenhausen and the conference organizers who displayed a warmth both during the congress sessions and afterwards. Visitors were delighted to see how beautiful the united Berlin has become with a wealth world class of tourist attractions.

The WAPM is grateful to Joachim Dudenhausen and his team for organizing this most successful meeting which will be long remembered by all who were fortunate enough to have participated in it.

Frank A. Chervenak, MD

President of the World Association of Perinatal Medicine 


\section{Awards}

The Erich Saling Award of the WAPM 2010 was awarded to two outstanding scientists: Prof. Anne Greenough, King's College Hospital, Department of Child Health, London, who gained the award for her excellence in neonatal work and research, and Prof. Joachim W. Dudenhausen, Charité, Berlin, who received the prize for his outstanding contribution to the fielt of obstetrics. A jury of selected scientists, chaired by Prof. Frank A. Chervenak, the President of the WAPM chose the recipients. The award ceremony was presided over by the founder of the prize himself, Prof. Erich Saling, who also spoke at the cermony.

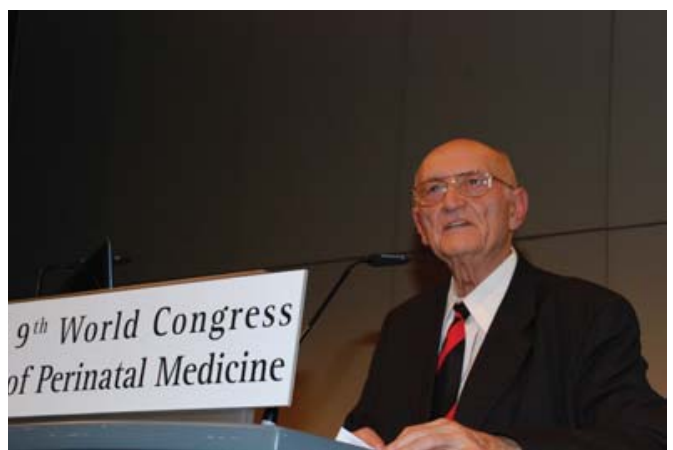

Prof. Erich Saling at the awarding ceremony. 